\section{MS13 O1}

Old tools for new materials Catalina Ruiz-Pérez, Jorge Pasán, Fernando S. Delgado, Oscar Fabelo, Laura Cañadillas-Delgado, María del Pino Déniz, Eliezer Sepúlveda. Laboratorio de Rayos $X$ y Materiales Moleculares, Dpto. Física Fundamental II, Facultad de Física, Universidad de La Laguna, Tenerife, Spain. E-mail: caruiz@ull.es

Keywords: Crystal engineering, coordination networks, molecular materials

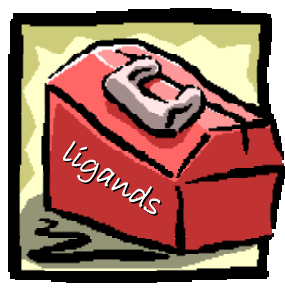

Making crystals by design is the paradigm of crystal engineering [1]. The goal of this field of research is that of assembling functionalized molecular and ionic components into a target network of supramolecular interactions [2]. This "bottomup" process generates collective supramolecular properties from the convolution of the physical and chemical properties of the individual building blocks with the periodicity and symmetry operators of the crystal [3].

One can envisage two main subareas of crystale engineering, namely of coordination networks and of molecular materials, even though all possible intermediate situations are possible. The preparation of coordination networks or polymers can be appropriately described as periodical coordination chemistry and exploit the possibility of divergent ligan-metal coordination, as opposed to the more traditional convergent coordination chemistry operated by chemating polydentate ligands.

While in periodical coordination chemistry it is useful to focus on the knots and spacers in order to describe the topology of the networks, when dealing with molecular materials what matters most are the characteristics of the component molecules or ions and the type of interactions holding these building blocks together.

[1] Desiraju, G.R., Crystal Engineering: The Design of Organic Solids, Elsevier, Amsterdam, 1989.

[2] Braga D., Grepioni, F., Acc. Chem. Res. 2000, 33, 601.

[3] Braga D., Grepioni, F., Orpen, A.G. Crystal Engineering: From Molecules and Crystals to Materials, Kluwer Academis Publishers, Dordrecht, 1999.

\section{MS13 O2}

Coordination networks based on carboxylate and 及-diketonate ligands Andrew D. Burrows, Department of Chemistry, University of Bath, UK.

E-mail: a.d.burrows@bath.ac.uk

Keywords: metal-organic frameworks, supramolecular chemistry, coordination complexes

The realisation that metal-organic frameworks (MOFs) can be robust, porous materials has led to an explosion of interest in these compounds. The first part of the presentation will focus on the synthesis of MOFs and demonstrate how the structure of the isolated product depends on factors such as solvent purity, temperature and $\mathrm{pH}$ [1]. Reactions of zinc(II), cadmium(II) and cobalt(II) with di- and tri-carboxylates will be described and the

structures obtained discussed. The second part of the presentation will concentrate on the use of non- carboxylate ligands in the synthesis of MOFs. One- and two-dimensional networks formed from bis( $\beta$-diketonate) ligands will be described, as will products formed from mixed-donor ligands containing a $\beta$-diketonate functionality in addition to a cyano or pyridyl group [2].

[1] Burrows, A. D., Cassar, K., Friend, R. M. W., Mahon, M. F., Rigby, S. P., Warren, J. E. CrystEngComm, 2005, 7, 548;

[2] Burrows, A. D., Cassar, K., Mahon, M. F. and Warren, J. E., Dalton Trans., 2007, in press.

\section{MS13 O3}

Crystal Engineering of Porphyrin Framework Solids

I. Goldberg, S. George, S. Muniappan, S. Lipstman, School of Chemistry, Sackler Faculty of Exact Sciences,

Tel-Aviv University, 69978 Tel-Aviv, Israel.

E-mail: goldberg@post.tau.ac.il

Keywords: crystal-engineering, framework solids, porphyrin coordination polymers

The chemical and structural diversity of porphyrins allows us to reasonably control their self-assembly process and to alter systematically the composition, topology, porosity and functionality of the supramolecular arrays that form.[1] The crystal engineering approach represents an attractive "bottom-up" strategy to tailoring ordered lattice materials and organic zeolite analogs from suitably functionalised tetraarylporphyrin building blocks.

In this work, targeted synthesis of framework coordination polymers was achieved by reacting the meso-substituted (3-carboxyphenyl)- and (4-carboxyphenyl)-porphyrin scaffolds with common salts of lanthanide metal ions.[2] The large size, high coordination numbers and strong affinity of the latter for oxo ligands, combined with favourable hydrothermal conditions, allowed the formation of open three-dimensional single-framework architectures by coordination poly-merisation. In the polymeric arrays the tetra-dentate porphyrin units are inter-coordinated in three dimensions by multi-nuclear assemblies of the bridging metal ions. The latter serve as construction pillars of the supramolecular arrays, affording stable structures.

These materials reveal several modes of polymerisation, which differ by the spatial functionality of the porphyrin building blocks, coordination patterns of the lanthanidecarboxylate assemblies and the topology of the resulting frameworks.[3] All structures exhibit, periodically spaced channel voids that perforate the polymeric architectures and are accessible to other molecular components.

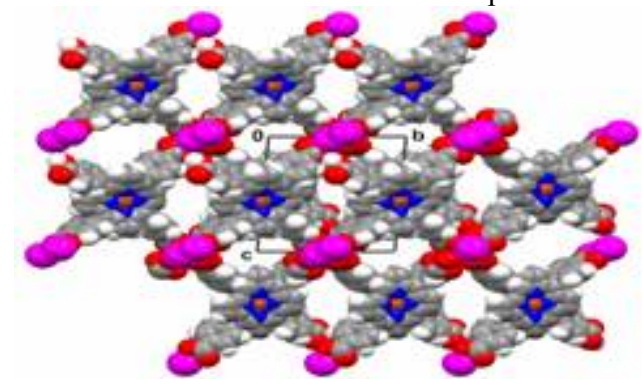

Typical view of the 3D polymeric structure down the coordination pillars.

[1] Goldberg, I., Chem. Commun. 2005, 1243-1254.

[2] George, S.; Lipstman, S.; Goldberg, I., Cryst. Growth Des., 2006, 6, 2651-2654. 